\title{
Research on Appendage SoftMan Based on Machine Cogmatics
}

\author{
Danfeng $\mathrm{Wu}^{1,2,3,{ }^{*}}$, Qingchuan Zhang ${ }^{1,2}, \mathrm{Xu}$ Zhang ${ }^{1,2}$ and Chaoen Xiao ${ }^{1,4}$ \\ ${ }^{1}$ School of Computer \& Communication Engineering, University of Science \& Technology Beijing, Beijing 100083, \\ China \\ ${ }^{2}$ Beijing Key Laboratory of Knowledge Engineering for Materials Science, Beijing 100083, China \\ ${ }^{3}$ School of Software, Liaoning Technical University, Huludao Liaoning 125105, China \\ ${ }^{4}$ Beijing Electronic Science and Technology Institute, Beijing 100000, China
}

\begin{abstract}
At present, the robot embedded system has some common problems as poor closure and dynamic evolution. Aiming at the situation, the paper focused on the improvement of robot embedded system, and set up architecture of robot system whose control center is Appendage SoftMan based on the syncretic mechanism of robot and SoftMan. The paper mainly paid attention to the research on Appendage SoftMan: used machine cogmatics theory as the guide to put forward the SoftMan knowledge and behavior model and the corresponding specification of conduct; constructed the architecture of Appendage SoftMan, and designed it based on the SoftMan knowledge and behavior model; materialized the theoretical research work as a nonlinear node detecting robot to make an application. Practice had proved that the robot system whose control center is Appendage SoftMan can implement the dynamic and real-time change of task, and its performance could not be affected.
\end{abstract}

Keywords: Robot embedded system, syncretic mechanism, knowledge and behavior model of SoftMan, Appendage SoftMan

\section{INTRODUCTION}

At present, standardization and network of robot controller as well as the PC-based network controller had become research hotspots. The overall trend of robotics research is from industrial robots to types of service robots so that the robots possess the diversity of form and function in order to meet the personalized needs of different users ${ }^{[1]}$. Therefore,a complete open and reconfigurable robot control system can be replaced and modified by the user according to the actual needs gradually become the aim of academic circles at home and abroad $^{[2,3]}$.

Goran Ferencet al. put forward a kind of real-time distributed robot control system based on CORBA protocols [4]. Rossmann [5], etc. presented a modular control system architecture based on multi-agent. Gilpin K et al. of MIT [6] designed a Miche lattice type modular robot in 2006 and realized the robot form of any shape. Sachiko Nakagawa et al. proposed a distributed service framework which can make all sorts of equipment and Internet services integrated through robot service network protocols [7]. The EPFL [8] Sprowitz A et al. set up a modular robot platform called Roombot based on the idea of the future intelligent furniture in 2009. Ernesto et al. [9] carried on research in the concept design, modeling and control of proportional differential of four degrees freedom robot manipulator for application in reconfigurable manufacturing system.

Domestic research on robot started relatively late, but obtained certain achievement in open reconfigurable robot research. Wu Xiangyang [10] et al. proposed a distributed architecture of robot controller to realize the seamless connection from the Internet to device at the bottom using Ethernet as the system bus and uniform communication protocol. Liu MingYao [11] et al. put forward a kind of robot control method based on multiple Agent. Zhang Yuhua [12] et al. proposed a system implementation scheme of hardware and software of a new type of modular reconfigurable robot HitMSR system. Cai Zixing [13], etc. proposed a design scheme of a kind of dynamic and reconfigurable Control system based on heterogeneous MAS theory. Aiming at Software environment for mobile robot, Wang Yongji [14] et al. proposed to apply the idea of the software component in the robot system to implement the tracking task from mobile robot to moving target .

Compared with traditional embedded robot control system which is too closed and difficult to reuse, this paper puts forward the syncretic mechanism of SoftMan ${ }^{[15]}$ and robot, makes the personification features and personification behaviors of SoftMan integrated into the control system of robot, and builds a new kind of robot embedded system architecture (The syncretic mechanism of SoftMan and robot is shown in Fig. 1). SoftMan system can send the specific 


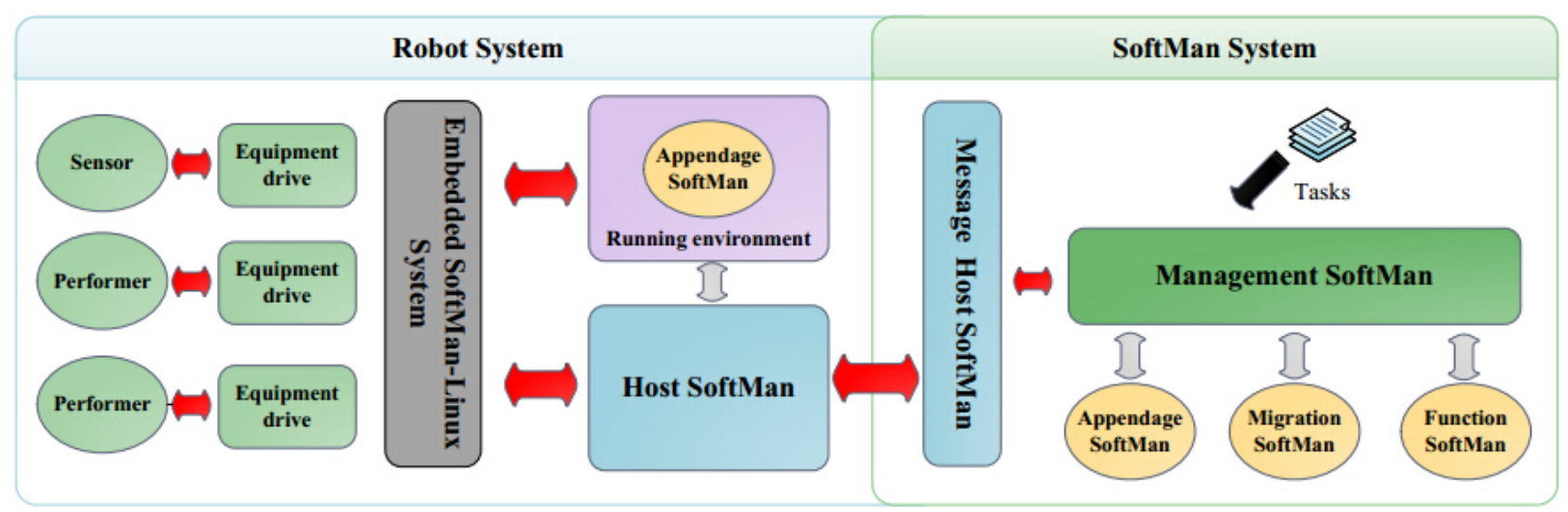

Fig. (1). The syncretic mechanism of SoftMan and robot.

Function SoftMan to the robot system according to different tasks and make this SoftMan the Appendage SoftMan attached to the robot system taking the place of original control function of robot. The Host SoftMan receives the migrated Appendage SoftMan and sets up its running environment to perform a task. When the task changed or Appendage SoftMan has update request, the robotic system can replace or update the Appendage SoftMan dynamically. By the migration and dynamic replacement of Appendage SoftMan, we can realize the real-time updates of the function and the task of the robot, shorten the upgrade cycle of control software of robot system.

Thus, Appendage SoftMan is the control center of robot embedded control system. As a result, this paper will focus on the study of Appendage SoftMan. On the basis of the definition of SoftMan, combined with machine learning model, SoftMan knowledge and behavior model is put forward; according to the Generalized Model theory in large system cybernetics and the Generalized Knowledge Representation, knowledge and behavior of SoftMan are described and specified; the structure of Appendage SoftMan is designed, and then on the basis of SoftMan knowledge and behavior model, the knowledge\&behavior unification description model is proposed, and service behaviors and task behaviors of Appendage SoftMan is designed and defined by the OMG IDL; the theoretical research is turned into the physical application finally.

\section{SOFTMAN KNOWLEDGE AND BEHAVIOR MOD- EL THEORY}

Machine Cogmatics is the study of the machine which has a certain "knowledge ability" [16, 17], and its theoretical core is "information - knowledge - intelligent conversion and unified theory". From the application of machine learning in Agent technology [18], natural language processing [19], emotional aspects [20], etc., we can realize the "virtual" robot, the SoftMan from the angle of the personification, survival in the network environment, its design and implementation should also follow related law of machine learning.

\subsection{SoftMan Knowledge and Behavior Model}

As a software entity to simulate extend and expand people's behavior and function, SoftMan should also follow the law of machine learning. However, in the "information knowledge - intelligent unified theory", they mainly research how the individual to get information, extract knowledge, converse and practice the smart strategy, but don't discuss the machine information processing through the coordination and collaboration in the group mode. And human learning capacity not only embodies in itself to "the understanding and transformation of the world, but also reflects in the way to know the world by sharing knowledge, transform the world by communication and corporation. Based on the research of SoftMan group [21], group information transformation model is proposed in this paper, as shown in Fig. (2).

From this model we can see, to solve the goal of given problem is a process of negotiation. First, because of individual ability is not the same, the processing of outside world information is different. When their insufficient access to information, they can share information with other entities and understand the different information. Second, the knowledge obtained in the process of cognitive access, not only has the inductive and deductive refined information, also, by taking share knowledge when their knowledge is not enough to make up for the defect of knowledge. At the same time, the intelligent strategy based on the knowledge transformation, likely will be able to perform through its own effect organs; may also be unable to complete alone, and need to cooperate with other entities to complete tasks.

Therefore, embarks from the group synergy goals, combined with machine learning and doing, SoftMan knowledge and behavior model (SKBM) can be defined as a triple, as shown in the definition 1.

\section{Definition 1}

\section{$S K M=<I, K B$, Behavior $>$}

Each tuple is defined as follows:

1) $I=\left\{I_{r e s} \cup I_{e}\right\}$ is the information SoftMan get or have. 


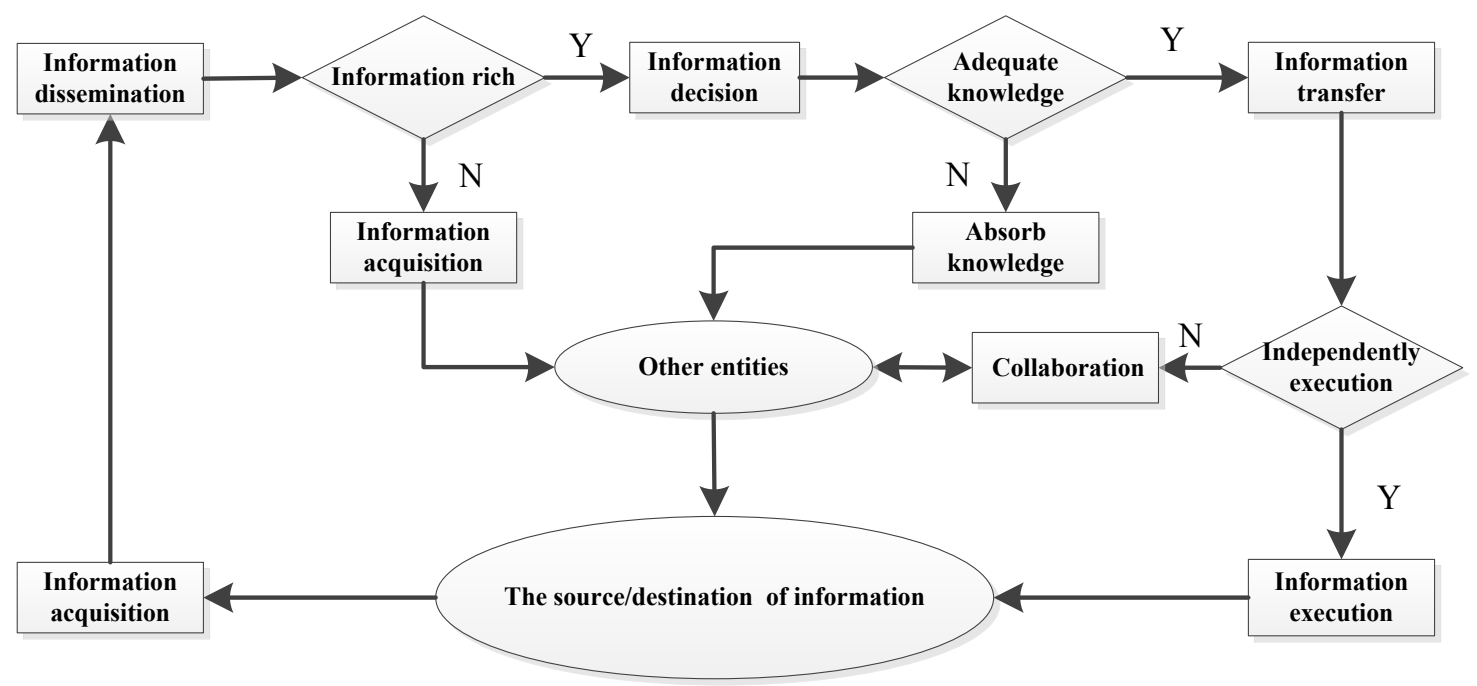

Fig. (2). Group information transformation model.

Among them, $I_{\text {res }}=\left\{\right.$ res $_{i}$, res $_{i}, \ldots$, res $\left._{n}\right\}(n>0)$ is the resources information set possessed by SoftMan .In knowledge learning, information is obtained from the outside world. In the group, SoftMan is not only the access to information, may also be other SoftMan (such as: coordinator) 's sources of information. Therefore, in order to let other SoftMan "know yourself", and get relevant information, the SoftMan needs describe its own resources.

$I_{e}=\left\{I_{\text {self }} \cup I_{\text {ext }}\right\}$ is a cognitive information collection, which contains the information get from the outside world ( $K B=\left\{K B_{\text {self }} \cup K B_{\text {ext }} \cup K B_{\text {share }}\right\}$ ) and its own message $\left(I_{\text {self }}\right)$.

2) $K B=\left\{K B_{\text {self }} \cup K B_{\text {ext }} \cup K B_{\text {share }}\right\}$ is a knowledge collection which is divided into two categories: knowledge by extracting and knowledge by sharing. Among them, $K B_{\text {self }} \cup K B_{\text {ext }}$ is the knowledge get through reasoning the external information and its own resource information. When SoftMan need work together to solve the problem, $K B_{\text {share }}$ is the knowledge derived from the problem for the group to share.

Furthermore, $\mathrm{KB}$ is defined as a triple: $K B=<$ Data,Type, Re lation $>$

Tuples are defined as follows:

(1) The Data is the data part of the knowledge of SoftMan, expressed as a triad: Data $=<$ DataType, DataContent, DataLen $>$.

(2) Type is the logic type of this SoftMan knowledge node, namely the resource type node or attribute type knowledge node.

(3) Relation is used to describe the fuzzy correlation between knowledge node and its children, expressed as Relation $=\bigcup<$ SubSKM $_{i}$, tValue $_{i}$, fValue $_{i}>\quad, \quad 0 \leq i \leq n$.
Among them, tValue $_{i}$ said the child knowledge node i's membership degree relative to this node, fValue $_{i}$ said the child knowledge node i's membership degree relative to this node.

3) Behavior $=\left\{\right.$ Behavior $_{\text {pro }}$, Behavior $_{\text {self }}$, Behavior $\left._{\text {req }}\right\}$ is the set of SoftMan behavior ability. It not only represents the SoftMan's processing power to the outside (Behavior ${ }_{p r o}$ ) and SoftMan's transform ability of information ( Behavior ), at the same time, also includes ability provided by otherSoftMan when they do coordination work.

Behavior $_{\text {self }}=\left\{f_{\text {resToe }}, f_{\text {eTTkb }}, f_{\text {kbTobeh }}\right\}$ is the information transformation mapping SoftMan have, $I_{\text {tran }}=\left\{f_{\text {resToe }}, f_{\text {eTokb }}, f_{\text {kbTobeh }}\right\}$. Among them:

$f_{\text {resToe }}: I_{\text {res }} \rightarrow I_{e}$ is the mapping from resource information to cognitive information;

$f_{\text {eTokb }}: I_{e} \rightarrow K B$ is the mapping from cognitive information to knowledge.

$$
\begin{gathered}
f_{\text {kbTobeh }}: K B \rightarrow\left\{\text { bev }_{1}, \text { bev }_{2} \cdots \text { bev }_{n}\right. \\
\left.\mid \text { bev }_{i} \in \text { Behavior }_{\text {pro }} \cup \text { Behavior }_{\text {req }}\right\}
\end{gathered} \text { is the mapping from }
$$

knowledge to intelligent strategy.

\subsection{The Description of SoftMan Knowledge \& Behavior}

In the SoftMan knowledge\&behavior model, knowledge is derived from the information resources, and is used to translate into smart strategy; behavior is the processing power to the outside resource based on intelligent strategy guidance. Obviously, the SoftMan based on the knowledge \& behavior model is a carrier integrating resources and services. 


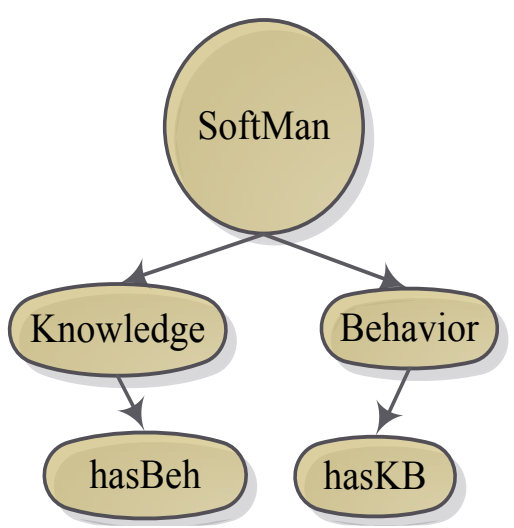

Fig. (3). Descriptive unification model of SoftMan knowledge\&behavior.

For a distributed system and based on the above description of the resources and services, this paper puts forward the unified way. In the network, resources is represented by one or one kind of SoftMan's knowledge, and resource access and control is supplied by the behaviors of SoftMan who represents the resources. So, when other SoftMan is demand for a resource, it is done through consultation with representatives of resources. Next, according to the "Generalized Model" theory of Large Scale System Cybernetics, based on the method of Generalized Knowledge Representation, we will descript and specify the knowledge \& behavior of SoftMan.

The unified model of knowledge\&behavior of SoftMan in distributed SoftMan system is shown in Fig. (3). SoftMan description includes two categories: knowledge and behavior. Knowledge build associated with behavior through" hasBeh", behavior build associated with knowledge through "hasKB".

\section{(1) The description of knowledge of SoftMan}

In the description of knowledge of SoftMan, we can use tree structure to organize the knowledge of SoftMan according to its field and its hierarchy, as shown in Fig. (4). SoftMan knowledge tree is composed of knowledge nodes and properties nodes.

The circle nodes in Fig. (4) are the SoftMan knowledge nodes, the specific attributes information of knowledge node is described by using the key-value pair. Each knowledge node can have multiple child knowledge nodes or multiple attributes nodes, and the name of the nodes is only. In the SoftMan knowledge tree, the knowledge nodes mainly play the role of a knowledge organization. They don't have attribute information and their information is described by child attribute nodes.

Square nodes in Figure are the SoftMan knowledge attribute nodes. An attribute node belongs to his father knowledge node, and each node of the attribute to the key value node to describe his father knowledge of specific information. Each attribute node using the key-value pair to describe the specific information of his father knowledge node
(2) The description and specification of behavior of SoftMan

From the view of cogmatics, SoftMan is an individual having certain information acquisition ability, able to extract knowledge from information, and can use the knowledge to reason, plan, and process the task requirements .From the point of personification, SoftMan is the cooperative entity under the condition of changing running environment and using environment, who can regulate its own behavior, organize and coordinate themselves to solve the problem. From the system abstract point, SoftMan is the software entity in the system which has both the functions of collaborative interaction and self management and reasoning. So, table 1 defines the specification of behavior of SoftMan to guide the implementation of prototype system.

Specifically, self-control type behaviors are used for the self- management of SoftMan, such as control own life, manage own resources. Synergy type behaviors are the related behaviors when SoftMan work together to solve the problem, such as communicate with other SoftMan, accept services provided by other SoftMan, provide self services to the outside, etc. And cognitive behaviors are the process of information - knowledge - intelligent strategy, the abilities of SoftMan to get the information into knowledge and acquire intelligent strategy based on the knowledge.

Among them, LifeCycleControl, ResourceControl, CommunicationBeh, CognitionBeh and MigrationBeh belong to natural behaviors. This part of the behaviors is all SoftMan required in the SoftMan system.

Behaviors for knowledge have input (in), output (out), relying on (required), and quality of service behavior supplied (QoS), etc. In view of the characteristics of behavior, there are certain ways in its description, as shown in Fig. (5). In the definition of behavior description, hasKB represents the associated knowledge. Info shows the related information of behavior, such as name of behavior, description of behavior, type of behavior, QoS of behavior, dependency on the environment of behavior, etc. Parameters indicate the related parameters among behavior runtime, such as input parameters, output parameters, etc. 


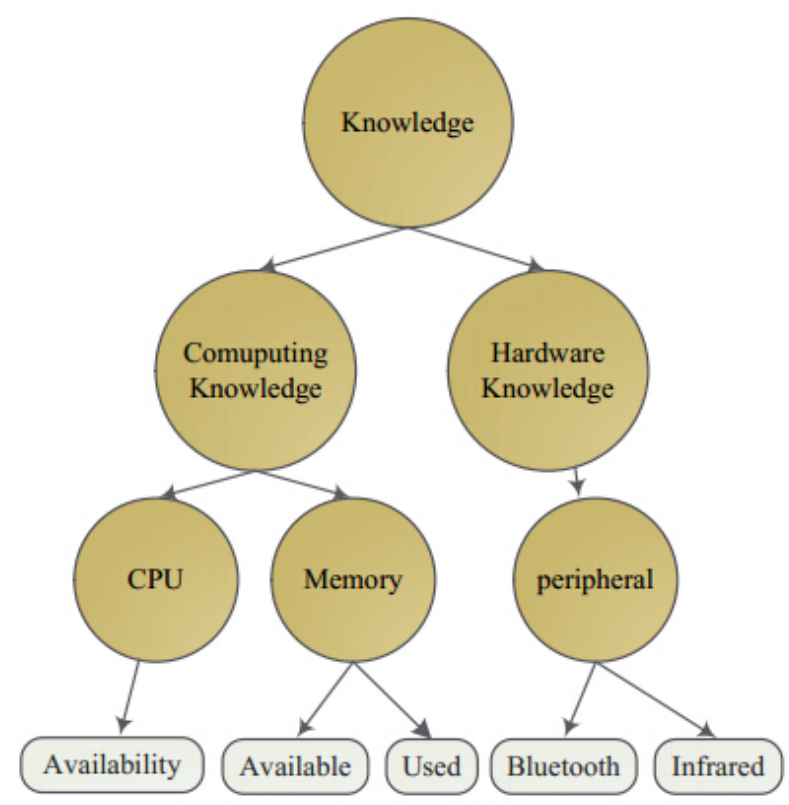

Fig. (4). SoftMan knowledge tree.

Table 1. The specification of behaviors of SoftMan.

\begin{tabular}{|c|c|c|}
\hline \multirow{2}{*}{ Self-control class } & Behavioridentifier & Behavior description \\
\hline \hline \multirow{2}{*}{ Synergyclass } & LifecycleControl & Resource Control Behavior \\
\cline { 2 - 3 } & ResourceControl Control Behavior \\
\hline \multirow{2}{*}{ cognition class } & CommunicationBeh & Communication Behavior \\
\cline { 2 - 3 } & SeviceBeh & Migration Behavior Behavior \\
\cline { 2 - 3 } & MigrationBeh & Knowledge extract and purification Behavior \\
\hline
\end{tabular}

\section{APPENDAGE SOFTMAN DESIGN}

\subsection{Architecture design of Appendage SoftMan}

In the robot control system, Appendage SoftMan is the control center, which is also the carrier of logic tasks and the final executor of user request, a professional SoftMan to complete the given task. Architecture design of Appendage SoftMan is shown in Fig. (6). In addition to self-behavior layer, Appendage SoftMan has the message processing layer, the migration preparing layer, the function calls layer and the task coordination layer.

\section{- Self-behavior layer}

Self-behavior layer refers to the SoftMan's own behaviors and reaction characteristics. Every SoftMan has this layer. SoftMan's own behaviors and reaction characteristics such as roaming itself, sleep, die young, die of old age and so on.
- Message processing layer

Message processing layer is mainly responsible for sending, receiving and dealing with messages. On the one hand, it makes self-requests as messages and sends to other SoftMan; on the other hand, it processes the receiving messages, and then makes corresponding reaction.

- Migration preparing layer

Migration preparing layer is responsible for sorting the knowledge base associated with current Appendage SoftMan, so that when task completed or request replace Appendage SoftMan, host SoftMan can package the knowledge base information of SoftMan and program, and deliver package to SoftMan system.

- Function calls layer

Function calls layer is the entrance to the tasks and management control of Appendage SoftMan. 


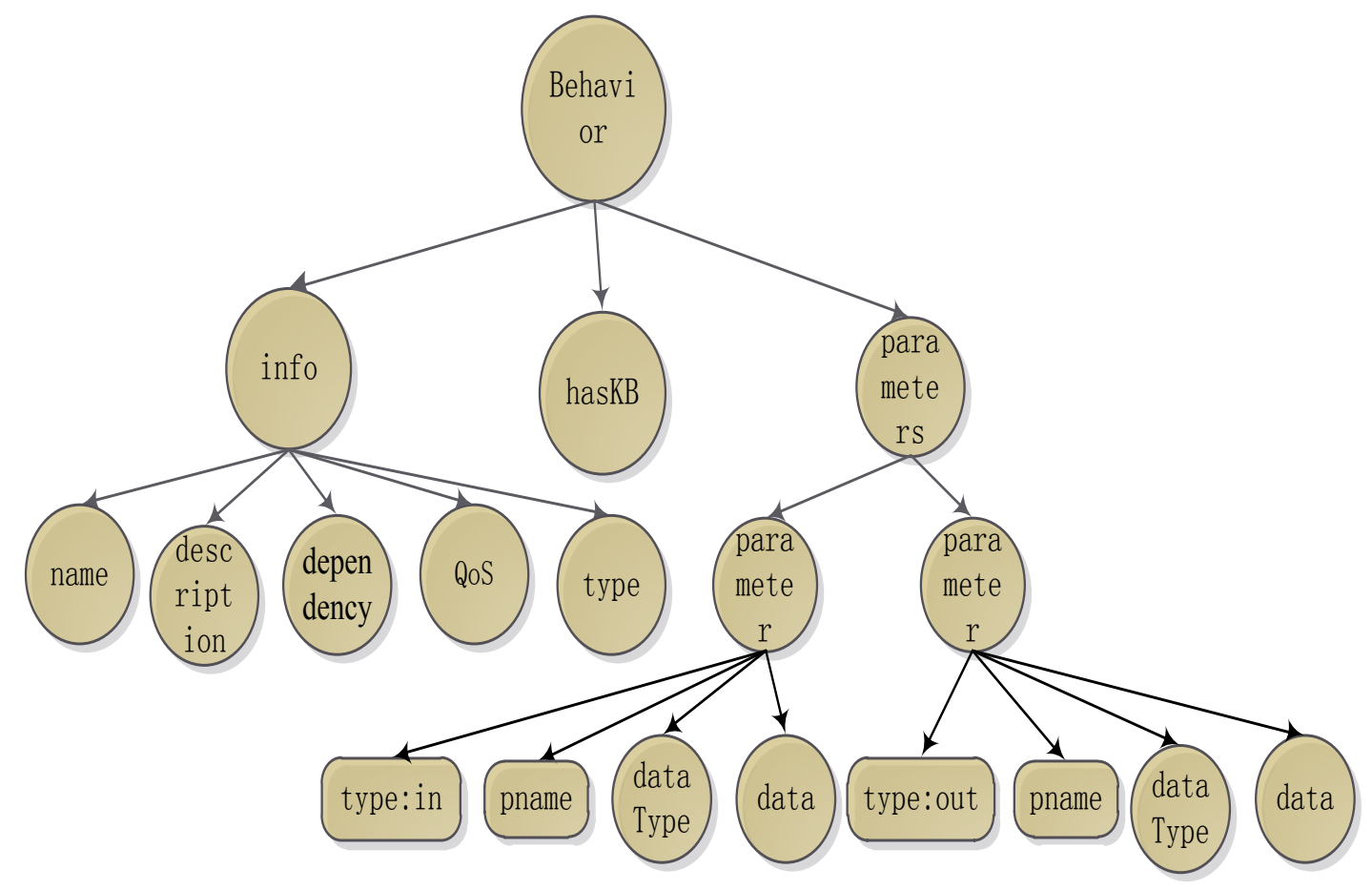

Fig. (5). SoftMan behavior description.

- Task coordination layer

When an Appendage SoftMan work with other Appendage SoftMan collaboratively, task coordination layer provides collaborative control mechanism and message buffer. Collaborative policies and instructions is transferred from Host SoftMan's collaborative control layer in robot or Management SoftMan's collaborative control layer in node endpoint.

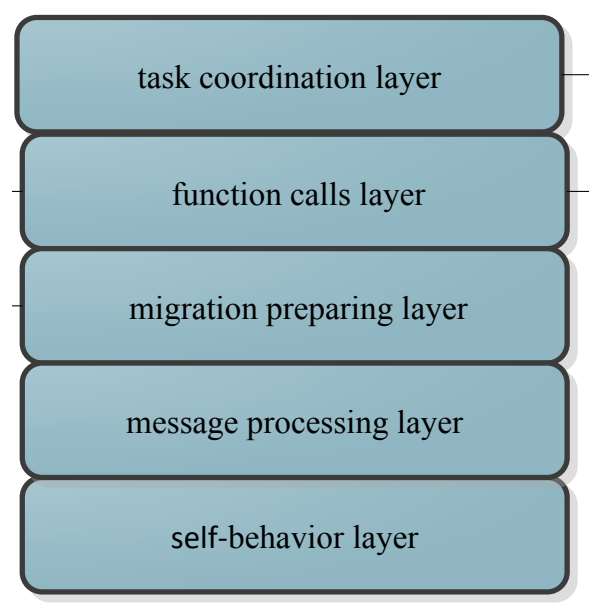

Fig. (6). Architecture of Appendage SoftMan.

\subsection{Descriptive Unification Model of Appendage Soft- Man Knowledge \& Behavior}

Applying Descriptive unification model of SoftMan knowledge \& behavior had been presented in section 1.2 to
Appendage SoftMan organization architecture and the abstract modeling of node data, and according to architecture design of Appendage SoftMan, we established the descriptive unification model of Appendage SoftMan knowledge \& behavior, as shown in Fig. (7).

In Fig. (7), white nodes are the common knowledge nodes, their name are defined by user; gray nodes are the reserved knowledge nodes, their name are immutable. The meaning of the reserved nodes is shown in Table 2.

Among them, Appendage SoftMan as a root node, represents an Appendage SoftMan, and includes of knowledge base (KnowLedgeBase) and behavior collection (Behaviors). Message, Runtime Environment, Context, and Task as the first-level child nodes of KnowLedgeBase, respectively, represents the knowledge involved in Appendage SoftMan, message processing layer, runtime support library, computing Context and the task coordination layer. Behaviors represent all behaviors of Appendage SoftMan, can be divided into life cycle control behaviors (LifeCycleCtrl), system resources control behaviors (ResCtrl), communication behaviors (CommBehs), cognition behaviors (CognBehs), migration preparing behaviors (MigBehs), service class behaviors (SrvBehs ) and extended service class behaviors (ExBehs).

By definition 1 we can see that solid line connecting in Fig. (8) indicates the precise relevance, tvalue is 1 , fvalue is 0 ; dotted line connecting indicates the fuzzy relevance, tvalue is less than 1, fvalue is greater than zero. Fun 1 and Fun2 in SrvBehs indicate Appendage SoftMan's services provided to external, and are marked with the solid line; the identification dotted line between ExBehs and Fun2 indicates the ascension of Fun2 behavior level which means that 


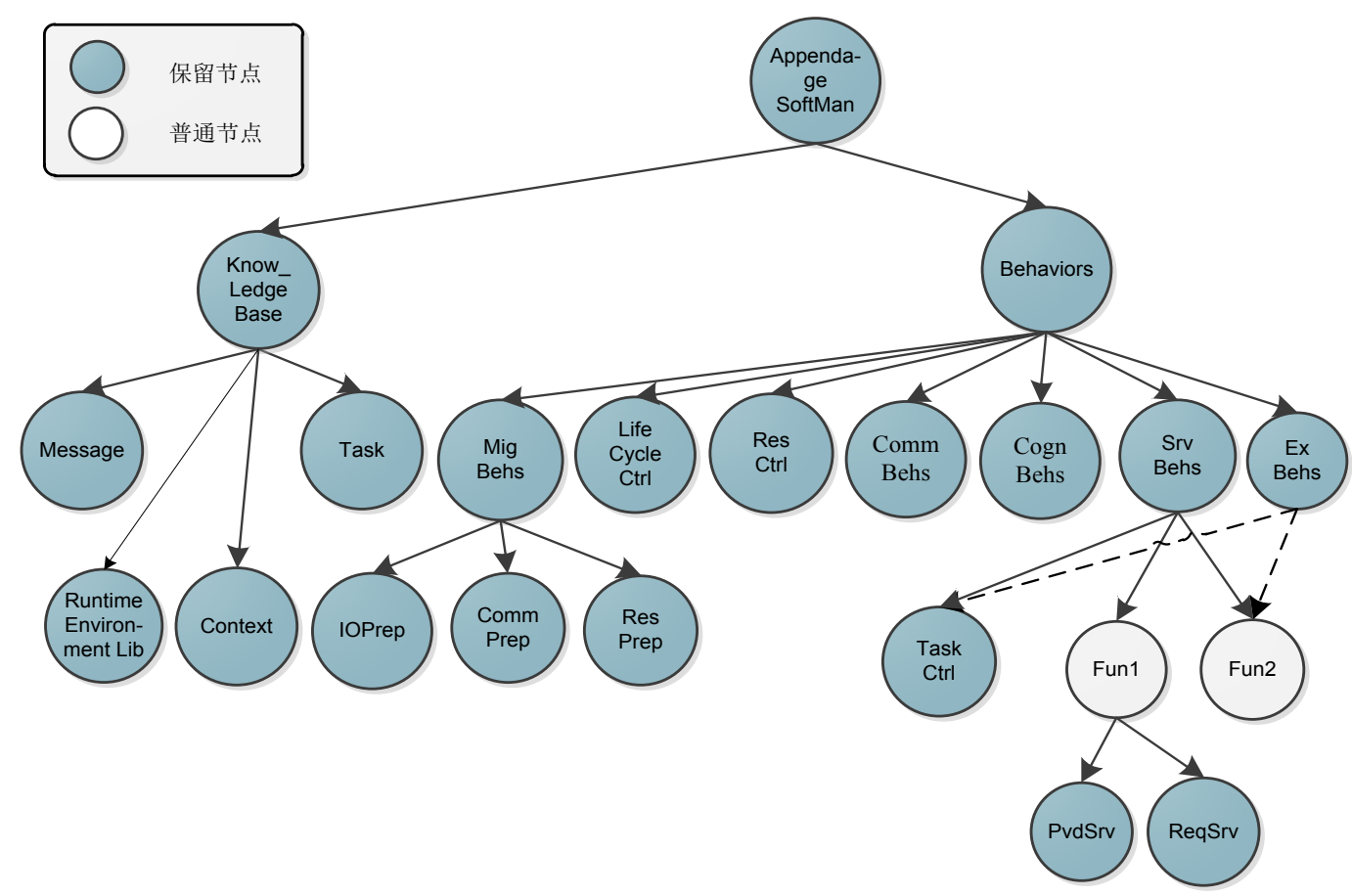

Fig. (7). Descriptive unification model of Appendage SoftMan knowledge \& behavior.

promoted to extended service behavior, and has higher priority at the time of behavior matching.

Appendage SoftMan mainly bear the business performing logic written by application developers, it has less system management behaviors. Therefore, in the construction process of Appendage SoftMan, the normative definition of service behaviors and task behaviors related with business is especially important. Clear and strict normative definition of conduct lays a good foundation for the interaction between Appendage SoftMan and other SoftMan.

\subsection{Appendage SoftMan Behavior Description}

In the behaviors of Appendage SoftMan described in figure7, MigBeh, LifeCycleControl, ResControl, CommBeh and CognBeh belong to its ontology behaviors, all SoftMan possess these behaviors introduced in section 1.2. Therefore, this section only elaborates SrvBeh and TaskCtr and gives them design specifications respectively using OMG IDL.

Table 2. Meaning of reserved nodes.

\begin{tabular}{|c|c|}
\hline 保留节点名称 & 意义 \\
\hline \hline AppendageSoftMan & Appendage SoftMan \\
\hline KnowLedgeBase & Appendage SoftMan knowledge base \\
\hline Message & Appendage SoftMan message \\
\hline RuntimeEnvironmentLib & runtime support library \\
\hline Context & Computing context information \\
\hline
\end{tabular}

\begin{tabular}{|c|c|}
\hline Task & Appendage SoftMan task \\
\hline Behaviors & Appendage SoftMan behaviors description \\
\hline LifeCycleCtrl & Life cycle control behaviors \\
\hline ResCtrl & Resources control behaviors \\
\hline CommBehs & Communication behaviors \\
\hline CognBehs & Cognition behaviors \\
\hline MigBehs & Migration preparing behaviors \\
\hline IOPrep & I/O Migration preparing \\
\hline CommPrep & Communication migration preparing \\
\hline ResPrep & Resources preparing \\
\hline SrvBehs & service class behaviors \\
\hline ExBehs & Extended service class behaviors \\
\hline PvdSrv & Provide servicesto external \\
\hline ReqSrv & Service requirements \\
\hline TaskCtrl & \\
\hline
\end{tabular}

\subsubsection{The Description of Service Behavior of Appendage SoftMan}

All the service behaviors of Appendage SoftMan constitute the SrvBehs, these service behaviors carry all the business logic of Appendage SoftMan, the service behavior model of Appendage SoftMan is shown in Fig. (8). 


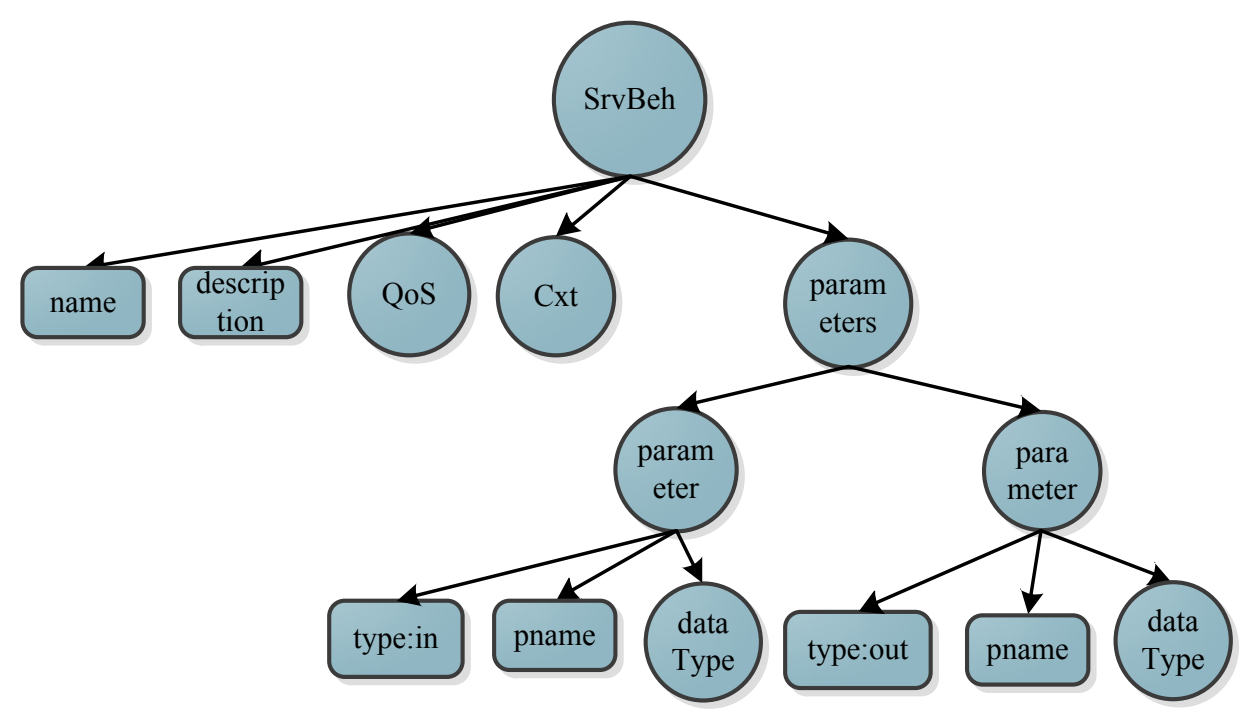

Fig. (8). Service behavior model of Appendage SoftMan.

Among them, attribute "name" represents the name of the service behavior; attribute "description" represents the description of service behavior; node "Qos" shows the description of service quality of service behavior; node "Cxt" expresses the computing context information; node "parameters" represents the parameter set of service behaviors; node "parameter" represents the description of a parameter; attribute "type" represents the parameter type, which is composed of input type "in" and output types "out"; attribute "name" represents the parameter name; node "data Type" represents the value type of parameter.

On the basis of the service behavior model, using OMG IDL, "SrvBeh" of Appendage SoftMan is defined as follows:

module ustb_softman_FunSM_api_Behavior\{

Import ustb.softman.api.Behavior.KnowledgeBase

InterfaceSrvBeh \{

intsetSrvBehName (in stringsrvBehName);

intsetSrvBehDesc (in stringdesc);

KnowledgeBasecreatPara(in string type, in string name, in stringdataType);

intaddPara(in KnowledgeBasepara );

intremoveParaByName(in string name );

intsetQos (in KnowledgeBaseqos);

intsetCxt (in KnowledgeBasecxt);

$\cdots$

\}

\}
Among them, setSrvBehName() is used to specify the name of function behavior; SetSrvBehDesc() is used to specify the description of function behavior; CreatPara() is used to create a parameter; AddPara()and ParaByName()is used respectively to add and remove a parameter; SetQos() is used to set the description of quality of service of functional behavior; $\operatorname{SetCxt()~is~used~to~set~the~computing~context~infor-~}$ mation that service behavior involved.

\subsubsection{The Description of Task Behavior of Appendage SoftMan}

In the expression of business logic, the description of task behavior is consistent with the description of service behavior of Appendage SoftMan, as shown in figure9.

In Fig. (9), node "value" represents the specific numerical value of parameter, the meaning of rest nodes is consistent with the service behavior model of Appendage SoftMan in section 3.2.1.

On the basis of above task behavior model, using OMG IDL, the task behavior "TaskCtrl" of Appendage SoftMan is defined as follows:

module ustb_softman_FunSM_api_Behavior\{

Import ustb.softman.api.Behavior.KnowledgeBase

InterfaceTaskCtrl \{

intsetSrvBehName (in stringsrvBehName);

intsetSrvBehDesc (in stringdesc);

KnowledgeBasecreatPara(in string type, in string name,

in stringdataType);

intaddPara(in KnowledgeBasepara ); 


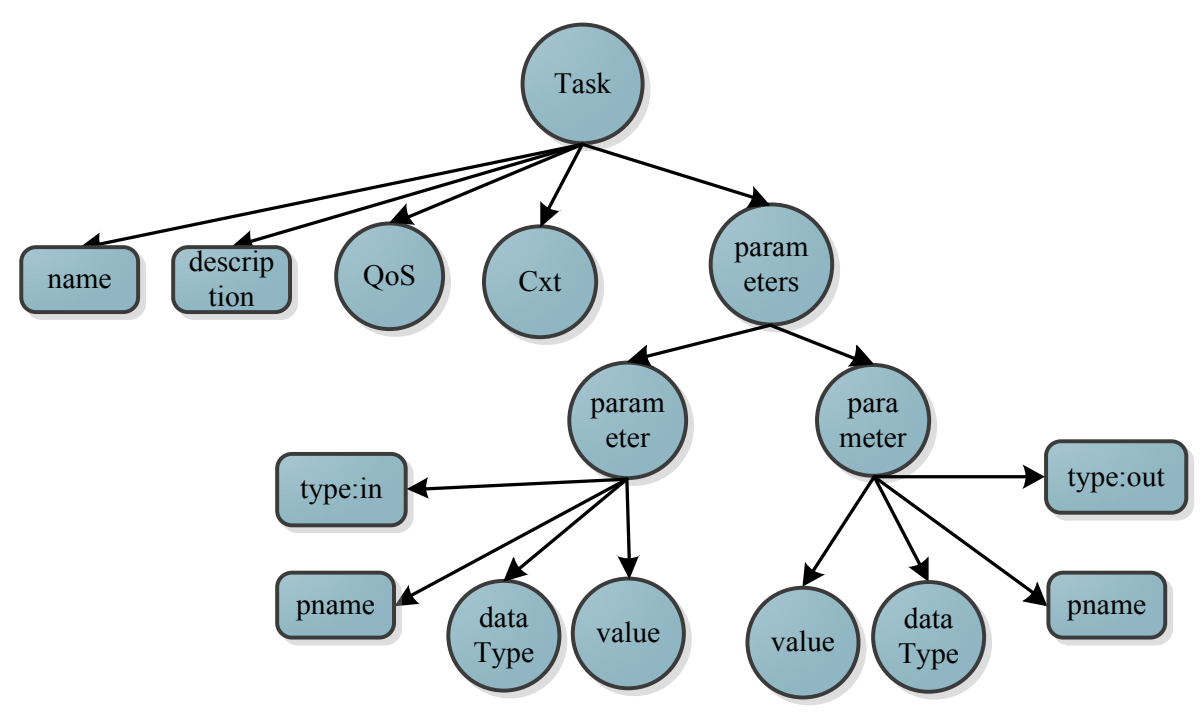

Fig. (9). The task behavior model of Appendage SoftMan.

intremoveParaByName(in string name );

intsetQos (in KnowledgeBaseqos);

intsetCxt (in KnowledgeBasecxt);

intsetValue(in string type, in KnowledgeBasevalue);

$\cdots$

\}

\}

Among them, setValue () is used to assign a value to the input parameter.

\section{APPLICATION CASE - THE DYNAMIC CONVER- SION OF FUNCTION OF A S-BAND BASED NON- LINEAR NODE DETECTOR}

At present, many detectors are emerging endlessly. When we put our focus on the mobile terminals of detectors, it is not difficult to find that: the update, modification, upgrade, maintenance, etc. involving in the function of detector can only be done by offline and static way because of the technical factors such as programming environment, implementation architecture and so on. However, from the perspective of syncretic mechanism of robot and SoftMan, we can convert the system of mobile terminal into a robot system which control center is Appendage SoftMan. The paper works eventually materialized a s-band based nonlinear node detecting robot, and the robot is a strongly dynamic and configurable open application.

Nonlinear Node Detector Runtime Architecture Exhibition Platform (NNRADEP) is shown in Fig. (10).

NNRADEP mainly consists of front-end detecting robot, data server and information management platform. The front-end detecting robot is located in the detecting area, responsible for testing and collecting data. It integrates into the LAN in the form through wifi and uploads the data to the data server; Data server and information management system are responsible for storing and managing the data which is reported by front-end detection robot, providing the convenient inquiry services. There are two kinds of nonlinear node: metal and electronic equipment, when detecting, robot needs switching between the two kinds of detecting objection. The update of control software adopts automatic migration method, namely, the robot needs which Appendage SoftMan, and the network platform will transport which Appendage SoftMan to the robot platform. The Host SoftMan will structure running environment for the new Appendage SoftMan so as to realize the dynamic replace of function of detecting robot, and the robot needn't to stop.

The SoftMan who perform detection function mainly include the Appendage SoftMan "CollectElectromagnetismInfo", Function SoftMan "ComputeDetectParameter" and Appendage SoftMan "DetectPNNode". Among them, CollectElectromagnetismInfo is responsible for collecting the surrounding electromagnetic environment information, and reporting information to ComputeDetectParameter; ComputeDetectParameter is responsible for calculating the detecting configuration parameter, and configurating DetectPNNode; DetectPNNode implements harmonic detection function circularly. The description of loading and configuring process of SoftMan is shown as follows:

1) Loading the Function SoftMan "ComputeDetectParameter" on the node endpoint;

2) Assigning the Appendage SoftMan "CollectElectromagnetismInfo" to the robot, collecting the surrounding electromagnetic environment information and reporting to ComputeDetectParameter;

3) Function SoftMan "ComputeDetectParameter" computes the configuration parameters of DetectPNNode based on the information of electromagnetic environment; 

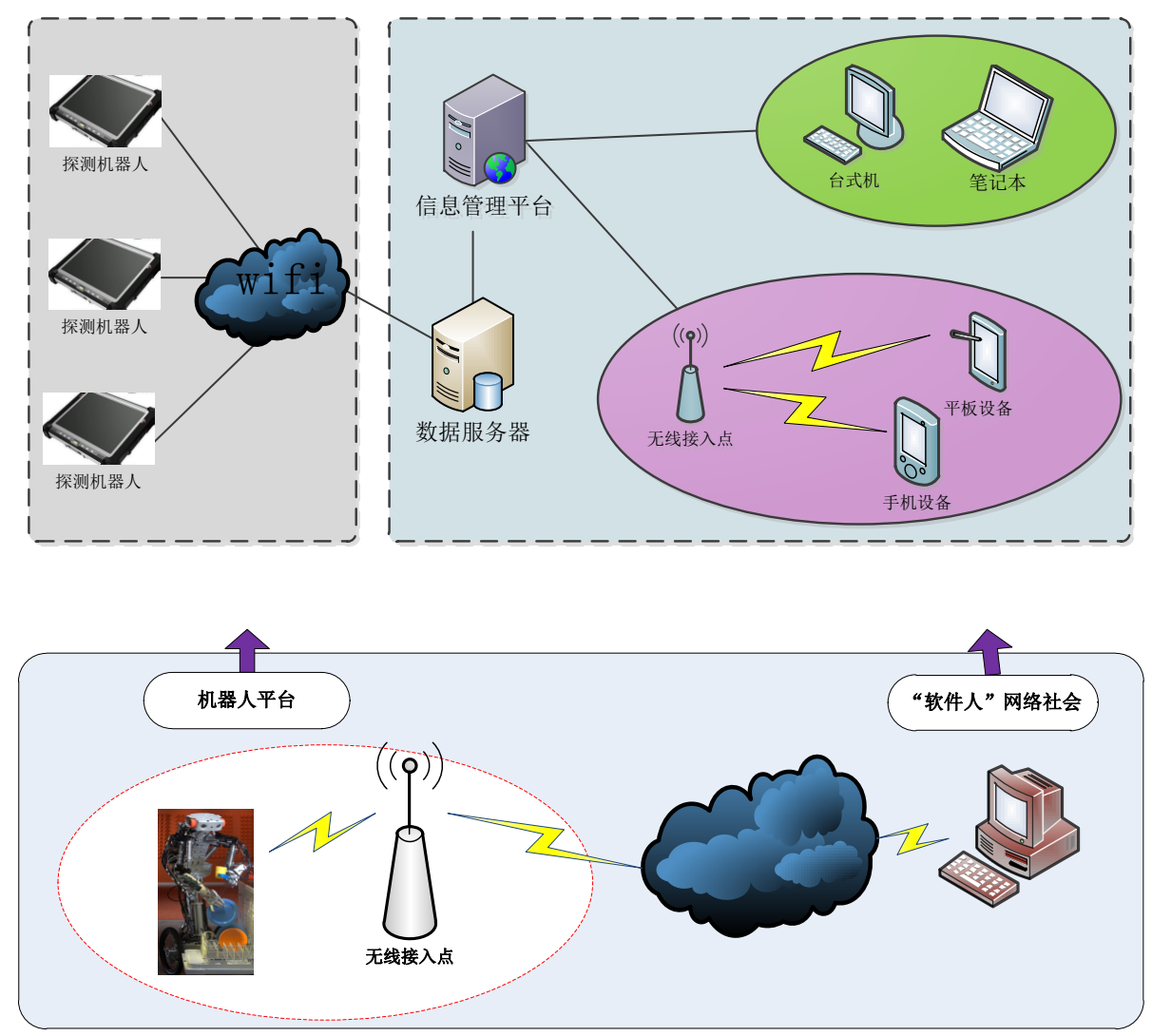

Fig. (10). Nonlinear Node Detector Runtime Architecture Exhibition Platform (NNRADEP).

4) Assigning DetectPNNode to the robot, and initializing the robot according to the configuration parameters given by ComputeDetectParameter;

5) DetectPNNode performs detecting function to finish nonlinear node detection task.

In the new environment, the system will repeat the above process and recalculate the configuration parameters.

\section{CONCLUSION}

In this paper, we proposed the syncretic thought of robot and SoftMan, transformed Function SoftMan which migrate from SoftMan platform to the control body of robot platform -Appendage SoftMan. Different Appendage SoftMan made robots have different function, so as to realize the seamless intelligent transformation between the robot functions. In the process of research of Appendage SoftMan, we used the theory of Machine Cogmatics, put forward the SoftMan knowledge and behavior model and the corresponding specification of conduct. On this basis, we finished the design of Appendage SoftMan and materialized the design results as a nonlinear node detection robot for practice. From a macro perspective, the robot system possessing the control center of Appendage SoftMan, had turned into a tangible SoftMan entity in logically, and activated in the physical space (i.e., incorporeal materialize), SoftMan system had become a mix of virtual and actual, larger artificial life system.

\section{CONFLICT OF INTEREST}

The authors confirm that this article content has no conflicts of interest.

\section{ACKNOWLEDGEMENTS}

Declared none.

\section{REFERENCES}

[1] Wang Tianmiao,Tao Yong,Chen Yang. Research and Development of Service Robotics. Chinese Science: Computer Science.2012.Vol.42 (9) : 1049-1066.

[2] Juan A. Fernanderz, Javier Gonazles. The NEXUS Open System for Integrating Robotic Software. Robotics and Computer Integrated Manufacturing, 1999,15:431-440

[3] Can Isik A A.Meystel. Pilot Level of a Hierarchical Controller for an Unmanned Mobile robot. In IEEE Journal of Robotics and Automation, 1998,4(3):241-255

[4] Goran Ferenc, Zoran Dimić, Maja Lutovac, Jelena Vidaković, Vladimir Kvrgić. Distributed robot control system implemented on the client and server PCs based on the CORBA protocol. Embedded Computing (MECO), 2012 Mediterranean Conference on, 1921 June 2012, pp. $158-161$

[5] E. Freund, R. Jurgen, M. Schluse, and C. Schlette, Using supervisory control methods for model based control of multi-agent systems, Robotics, Automation and Mechatronics Conference on IEEE, 2004.

[6] Gilpin K, Kotay K, Rus D, et al. Miche: modular shape formation by self-disassembly. Int J Robot Res, 2008, 27:345-372

[7] Sachiko Nakagawa, Noboru Igarashi, Yosuke Tsuchiya, Masahiko Narita and Yuka Kato. An implementation of a distributed service 
framework for cloud-based robot services. Proceedings of IECON 2012 - 38th Annual Conference on IEEE Industrial Electronics Society, 25-28 Oct. 2012, pp.4148 - 4153

[8] Sprowitz A, Pouya S, Bonardi S, et al. Roombots: reconfigurable robots for adaptive furniture. IEEE Comput IntellMag, 2010, 5: 2032

[9] Ernesto, H, Pedro, J.O.Modelling and PD control of a novel reconfigurable robotic machining manipulator. AFRICON, 2011:1- 6

[10] WuXiangyang, Dai Xianzhong, MengZhengda. Researchondistributedarchitectureof robot controller. Journal of Southeast University(Natural Science Edition), 2003, 33(9):200-204

[11] Liu Mingyao, Tan Yuegang, Zhou Zude, Hu Yefa. A Distribution Control Algorithm for Reconfigurable Modular Robots. Journal of WUT (Information \&Management Engineering) ,2005,27(1):6-9.

[12] Zhang Yuhua,Zhao Jie,Zhang Liang, et al.Novel Modular Selfreconfigurable Robot System.Chinese Journal of Mechanical Engineerng.,2006.05:175-178.

[13] Xie Feng, Xiao Xiaoming, Cai Zixing.On Reconfigurable Control System for Mobile Robot. Control Engineeringof China,2007,15(5):535-539.

[14] Liu Guocheng, Wang Yongji, Xu Jiansheng. Development of a Component-based Mobile Robot Software System. Robot. 2007,29(4):337-342
[15] Zeng Guang-Ping, TU Xu-Yan ,WANG Hong-Bo,,SoftMan Research and Applications. Science Press,2007

[16] Y X Zhong. An introduction to cogmatics: theory of transforms from information to knowledge and further to intelligence[J]. Chinese Engineering Science, 2004,(6)6:1-8.

[17] Zhong Yixing,The Principle of Machine Cogmatics:The Theory of Transformation and Unification of Information,Knowledge and Intelligence. Science Press, 2007

[18] Zhang Pengfei.Comprehensive-Information-Based Formal Pragmatic Approach For Agent Communication.Beijing University of Posis and Telecommunications, 2007.

[19] Y X Zhong. Comprehensive Information Based Methodology for Natural Language Understanding [J]. Journal of Beijing University of Posts and Telecommunications, 2004,27(4):1-12.

[20] Jiang, Pu.The comprehensive information emotion theory - An analysis on the framework[C].2nd International Conference on Information Engineering and Computer Science, ICIECS 2010, IEEE Computer Society,2010:1-4

[21] WANG Hong-Bo,ZENG Guang-Ping,WANG Zong-Jie,TU XuYan,SoftMan Group Intelligent Autonomous Coordination Model and Its Application.ACTA AUTOMATIC SINICA, 2007,33(9):924-929.

(C) Wu et al.; Licensee Bentham Open.

This is an open access article licensed under the terms of the Creative Commons Attribution Non-Commercial License (http://creativecommons.org/licenses/by-nc/3.0/) which permits unrestricted, non-commercial use, distribution and reproduction in any medium, provided the work is properly cited. 